\title{
Double-helix supramolecular nanofibers assembled from negatively curved nanographenes
}

Kenta Kato, ${ }^{1, \dagger}$ Kiyofumi Takaba, ${ }^{2, \dagger}$ Saori Maki-Yonekura, ${ }^{2, \dagger}$ Nobuhiko Mitoma, ${ }^{1,3,4}$ Yusuke Nakanishi, ${ }^{5}$ Taishi Nishihara, $, 13,6$ Taito Hatakeyama, ${ }^{7}$ Takuma Kawada, ${ }^{7}$ Yuh Hijikata, ${ }^{1,8,9}$ Jenny Pirillo, ${ }^{8,9}$ Lawrence T. Scott, ${ }^{10}$ Koji Yonekura, ${ }^{2,11, *}$ Yasutomo Segawa, ${ }^{1,3,12,13, *}$ and Kenichiro $\operatorname{Itami}^{1,3,8, *}$

\section{Affiliations:}

${ }^{1}$ Graduate School of Science, Nagoya University, Nagoya 464-8602, Japan

2 Biostructural Mechanism Laboratory, RIKEN, SPring-8 Center, 1-1-1 Kouto, Sayo, Hyogo 679-

5148, Japan

3 JST, ERATO, Itami Molecular Nanocarbon Project, Nagoya University, Nagoya 464-8602, Japan

${ }^{4}$ RIKEN Center for Emergent Matter Science, Wako 351-0198, Japan

${ }^{5}$ Graduate School of Science, Tokyo Metropolitan University, Hachioji 192-0397, Japan

${ }^{6}$ Institute of Advanced Energy, Kyoto University, Uji, Kyoto 611-0011, Japan

${ }^{7}$ Central Research Laboratory Technology and Development Division, Kanto Chemical Co., Inc., Saitama 340-0003, Japan

${ }^{8}$ Institute of Transformative Bio-Molecules (WPI-ITbM) Nagoya University, Nagoya 464-8602, Japan

9 Institute for Chemical Reaction Design and Discovery (WPI-ICReDD), Hokkaido University, Sapporo, Hokkaido 001-0021, Japan

${ }^{10}$ Department of Chemistry, University of Nevada, Reno, NV 89557-0216, USA

11 Advanced Electron Microscope Development Unit, RIKEN-JEOL Collaboration Center, RIKEN Baton Zone Program, 1-1-1 Kouto, Sayo, Hyogo 679-5148, Japan

${ }^{12}$ Institute for Molecular Science, Myodaiji, Okazaki, 444-8787, Japan

13 Department of Structural Molecular Science, SOKENDAI (The Graduate University for Advanced Studies), Myodaiji, Okazaki, 444-8787, Japan

* Correspondence to: yone@spring8.or.jp (K.Y.), segawa@ims.ac.jp (Y.S.), itami@chem.nagoyau.ac.jp (K.I.)

$30 \quad \uparrow$ These authors contributed equally.

\section{Abstract:}

The layered structures of graphite and related nanographene molecules play key roles in their physical and electronic functions. However, the stacking modes of negatively curved nanographenes remains unclear, owing to the lack of suitable nanographene molecules. Herein we report the synthesis and one-dimensional supramolecular self-assembly of negatively curved nanographenes without any assembly-assisting substituents. This curved nanographene selfassembles in various organic solvents and acts as an efficient gelator. The formation of nanofibers was confirmed by microscopic measurements, and an unprecedented double-helix assembly by continuous $\pi-\pi$ stacking was uncovered by three-dimensional electron crystallography. This work not only reports the discovery of an all-sp ${ }^{2}$-carbon supramolecular $\pi$-organogelator with negative curvature, but also demonstrates the power of three-dimensional electron crystallography for the structural determination of submicrometer-sized molecular alignment. 


\section{Main Text:}

The layered structures of graphite and related nanographene molecules play a key role in their physical and electronic properties (1-6). The well-ordered molecular alignment of nanographenes and its structural determination are of interest in order to gain insight into a variety of carbon materials. It is well known that the one-dimensional (1D) assembly of planar nanographenes (e.g. hexa-peri-hexabenzocoronene) can be achieved by introducing suitable peripheral substituents that tune solubility (Figure 1A) (7). The 1D assembly of bowl-shaped nanographenes was also achieved by convex-concave $\pi-\pi$ stacking along with non-covalent interactions $(8-10)$. However, the stacking modes of negatively curved nanographenes remains unclear, owing to the lack of suitable nanographene molecules. In 2013, our group reported warped nanographene (WNG), a large nonplanar nanographene containing five seven-membered rings (Figure 1C) (11). In spite of its large structure with 80 carbon atoms $\left(\mathrm{C}_{80} \mathrm{H}_{30}\right)$, WNG is soluble in various organic solvents because its negative curvature hinders $\pi-\pi$ stacking. During related works on WNGs (12-15), we found that a synthetic intermediate of WNG (1-biph) efficiently forms stacked $\pi-\pi$ dimers in the crystalline state (12). Considering the high solubility of the WNG family and the existence of a $\pi$ $\pi$ stacking mode, we hypothesized that substituent-free $1 \mathrm{D}$ assemblies could be realized by using negatively curved nanographenes (Figure 1B).

A

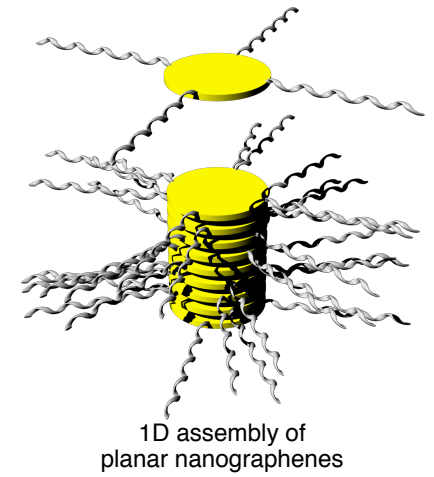

C

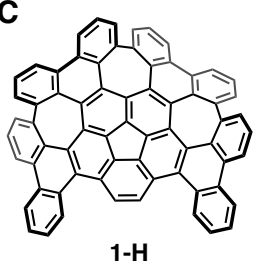

1D-assembling negatively curved PAH this work

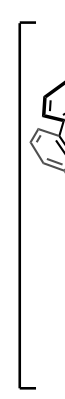

B
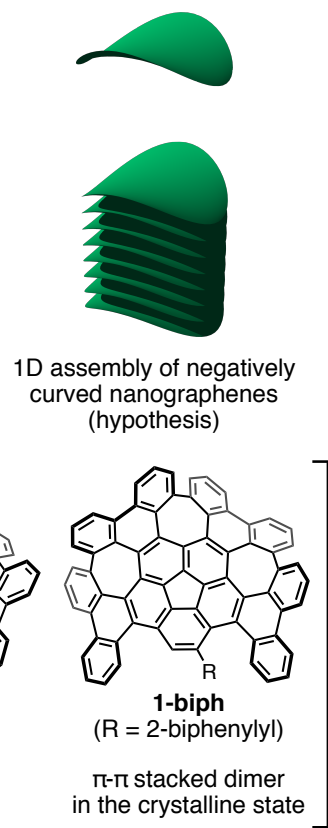

Figure 1. 1D assemblies of nanographenes. (A) Assembly of planar nanographenes with peripheral substituents. (B) Assembly of negatively curved nanographenes (hypothesis). (C) Structures of warped nanographene (WNG) and its analogues (1-H, 1-biph).

Herein, we report the synthesis and 1D self-assembly of a newly designed nanographene 1-H $\left(\mathrm{C}_{68} \mathrm{H}_{28}\right)$, a negatively curved PAH with 12 carbon atoms fewer than WNG (Figure 1C). Serendipitously, we discovered that 1-H self-assembles in various organic solvents and works as a highly efficient gelator that forms organic gels at concentrations of $<1 \mathrm{wt} \%$. Transmission 
electron microscopy (TEM) and atomic force microscopy (AFM) measurements confirm that 1-H forms fibers with diameters of $\sim 2.8 \mathrm{~nm}$. The presence of efficient $\pi-\pi$ interactions in the fiber structures is supported by a bathochromic shift in the fluorescence spectrum of the gel state relative to that of dilute solutions of 1-H. Finally, using three-dimensional (3D) electron crystallography, the double-helix $\pi-\pi$ stacking mode of $\mathbf{1 - H}$ in the supramolecular nanofiber was revealed.

The synthesis of 1-H was accomplished by a synthetic route analogous to that used to prepare WNG as shown in Figure 2 (11). We selected tetrakis(biphenyl-2-yl)corannulene (2) as the precursor of 1-H. Fourfold Suzuki-Miyaura coupling of a regioisomeric mixture of tetraborylcorannulene 3 (16) with 2-bromobiphenyl afforded 2. A Scholl reaction (17,18) of 2 was brought about by the action of 2,3-dichloro-5,6-dicyano- $p$-benzoquinone (DDQ) and $\mathrm{CF}_{3} \mathrm{SO}_{3} \mathrm{H}$ to generate $1-\mathrm{H}$ as a yellow solid in $56 \%$ yield based on 3 . The ${ }^{1} \mathrm{H}$ NMR and ${ }^{13} \mathrm{C}$ NMR spectroscopic analysis of 1-H suggest a $C_{2}$-symmetric structure in solution, and high-resolution mass spectra confirm a molecular formula of $\mathrm{C}_{68} \mathrm{H}_{28}$ for $\mathbf{1}-\mathbf{H}$.

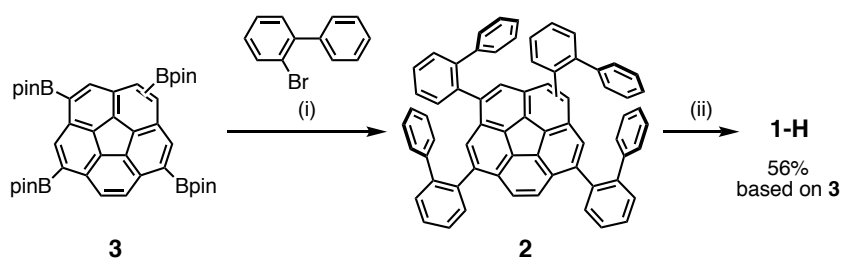

15 Figure 2. Synthesis of 1-H. Reaction conditions: (i) $\mathrm{Pd}_{2}(\mathrm{dba})_{3} \cdot \mathrm{CHCl}_{3}, \mathrm{SPhos}_{3} \mathrm{~K}_{3} \mathrm{PO}_{4}$, toluene/water, $100{ }^{\circ} \mathrm{C}, 24 \mathrm{~h}$; (ii) DDQ, $\mathrm{CF}_{3} \mathrm{SO}_{3} \mathrm{H}, 0{ }^{\circ} \mathrm{C}, 3 \mathrm{~h}$. Abbreviations: DDQ = 2,3-dichloro5,6-dicyanobenzoquinone; Bpin = 4,4,5,5-tetramethyl-1,3,2-dioxaborolan-2-yl; $\mathrm{dba}=$ dibenzylideneacetone; SPhos = 2-dicyclohexylphosphino-2',6'-dimethoxybiphenyl.

During recrystallization experiments, it was discovered that $\mathbf{1 - H}$ works as an efficient organogelator. When a $\mathrm{CH}_{2} \mathrm{Cl}_{2}$ solution of $\mathbf{1 - H}$ was slowly evaporated over the course of 1 day, an organogel was formed (Figure 3A,B). To the best of our knowledge, there is no report of any unfunctionalized aromatic hydrocarbon that works as an organogelator (19-26). Intrigued by this unique behavior, we conducted a detailed investigation of the gelating ability of 1-H in various organic solvents (Figure 3C). Some chloroalkanes and aromatic solvents yield transparent gels at low critical gelation concentrations (CGC; $0.2-0.8 \mathrm{wt} \%$ ), indicating the high gelation efficiency of 1-H. Slow diffusion of pentane vapor into solutions of $\mathbf{1 - H}$ in tetrahydrofuran (THF) and 1,1,2,2-tetrachloroethane (TCE) affords translucent gels with slightly higher CGCs (2.3 and 1.8 $\mathrm{wt} \%$, respectively) reflecting the high solubility of $\mathbf{1 - H}$ in THF and TCE. In hydrocarbons and polar solvents (triethylamine, acetonitrile, alcohols, dimethyl sulfoxide, and water), no gels are formed. In no case could single crystals of $\mathbf{1 - H}$ be obtained. This behavior stands in sharp contrast to the structurally similar derivative $\mathbf{1 - C l}$, which readily forms single crystals (for details, see Supplementary Materials (SM)). 
A

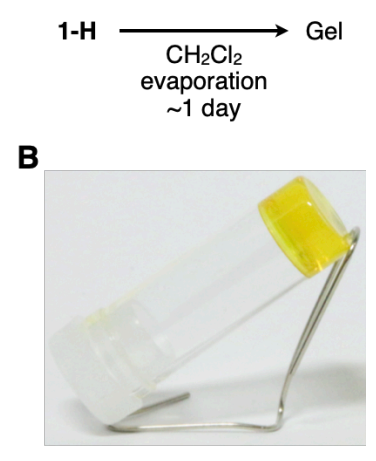

$\mathbf{E}$

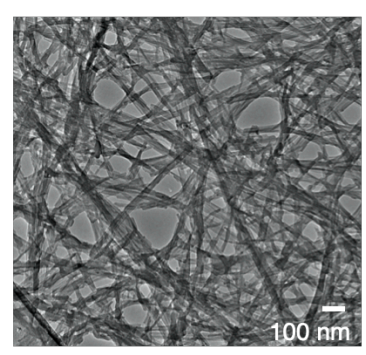

C

\begin{tabular}{|c|c|c|}
\hline Solvent & State $^{\mathrm{c}}$ & CGCa $[w t \%]$ \\
\hline $\mathrm{CH}_{2} \mathrm{Cl}_{2}$ & Gel & 0.3 \\
\hline $\mathrm{CHCl}_{3}$ & Gel & 0.3 \\
\hline $\mathrm{CCl}_{4}$ & Gel & 0.2 \\
\hline Benzene & Gel & 0.2 \\
\hline Toluene & Gel & 0.8 \\
\hline $\mathrm{CS}_{2}$ & Gel & 0.4 \\
\hline $\mathrm{C}_{2} \mathrm{H}_{2} \mathrm{Cl}_{4}$ & Gel & 1.8 \\
\hline THF & Gel & 2.3 \\
\hline Pyridine & $J^{b}$ & - \\
\hline Piperidine & $\mathrm{Jb}$ & - \\
\hline
\end{tabular}

$\mathbf{F}$

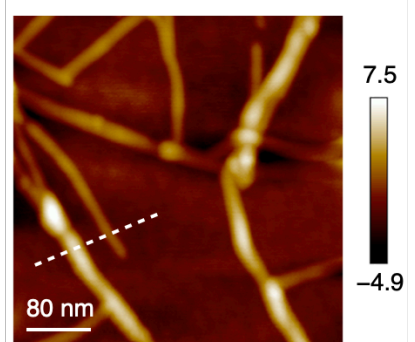

D
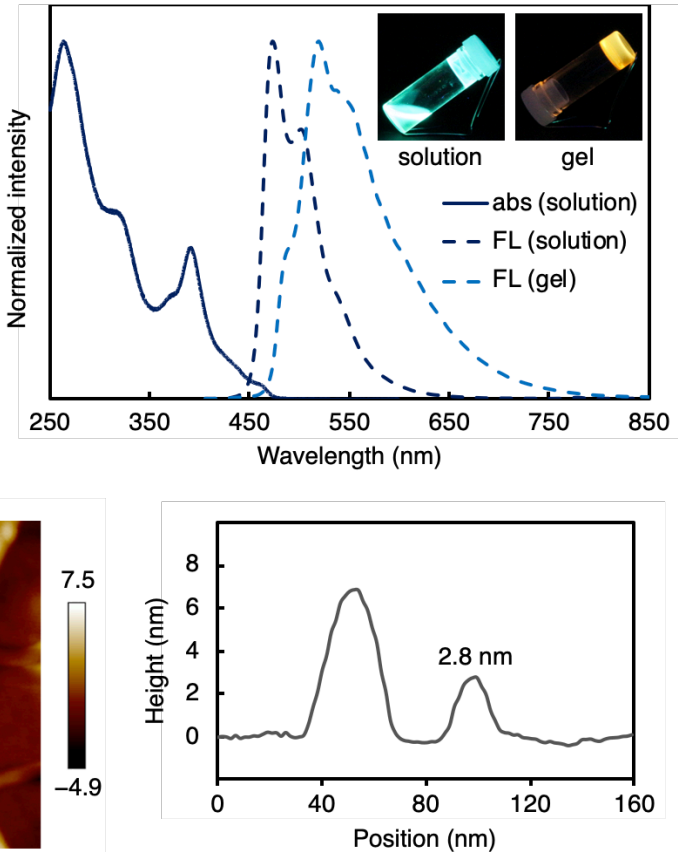

Figure 3. Gel formation of 1-H. (A) Gelation of 1-H. (B) Photo of the yellow organogel obtained by partial evaporation of a solution of $\mathbf{1 - H}$ in $\mathrm{CH}_{2} \mathrm{Cl}_{2}$. (C) Gelation properties of $\mathbf{1 - H}$ in organic solvents. ${ }^{a}$ CGC: critical gelation concentration. ${ }^{b} \mathrm{~J}=$ jelly precipitate. (D) A UV-Vis absorption spectrum (abs; solid line) and fluorescence spectra (FL; dotted lines) of $\mathbf{1 - H}$ in $\mathrm{CH}_{2} \mathrm{Cl}_{2}$ and in the gel state. The inserted photographs show the solution (left) and gel (right) under irradiation with UV light $(\lambda=365 \mathrm{~nm})$. Fluorescence spectra were recorded upon excitation at $350 \mathrm{~nm}$ (solution) or $390 \mathrm{~nm}$ (gel). (E) A TEM image of a dried gel obtained from a $\mathrm{CH}_{2} \mathrm{Cl}_{2}$ solution of 1-H. (F) An AFM image of a dried gel obtained from a $\mathrm{CH}_{2} \mathrm{Cl}_{2}$ solution of $\mathbf{1 - H}$ and the cross-sectional height profile taken along the dotted line in the AFM image.

The photophysical properties of 1-H support the existence of intermolecular $\pi$ - $\pi$ interactions in the gel state. In solution $\left(\mathrm{CH}_{2} \mathrm{Cl}_{2}\right)$, 1-H shows absorption maxima at 264, 320, 369, 390, and $460 \mathrm{~nm}$ and exhibits blue fluorescence, with peaks at 475 and $502 \mathrm{~nm}$ and a low fluorescence quantum yield $\left(\Phi_{\mathrm{F}}=0.20\right)$ upon excitation at $350 \mathrm{~nm}$ (Figure 3D). These results in solution are similar to those obtained for WNG (11). By contrast, in the gel state, 1-H exhibits yellow fluorescence, with the longest wavelength fluorescence peaks shifted bathochromically to $489 \mathrm{~nm}$ and $521 \mathrm{~nm}$ (Figure 3D). These aggregation-dependent changes in the fluorescence spectrum signify strong $\pi-\pi$ interactions among the molecules of $\mathbf{1 - H}$ in the gel state (27-29).

In imaging experiments, the fibrous structure of 1-H in the organogel was observed using both TEM and AFM (Figure 3E,F). The TEM image shown in Figure 3E establishes that 1-H forms nanofiber structures in the gel state. The diameter of the thinnest fiber determined by the crosssectional height profile in the AFM image (Figure 3F) measures ca. $2.8 \mathrm{~nm}$.

Finally, the nanofiber structure was fully analyzed by 3D electron crystallography. Needleshaped microcrystals of 1-H were collected from the supernatant of the gel obtained from a TCE solution at room temperature. As shown in Figure 4A, the needle-shaped microcrystals were found 
by TEM measurement to have widths of around $0.5 \mu \mathrm{m}$. Electron diffraction patterns were recorded at a specimen temperature of $\sim 98 \mathrm{~K}$ (Figure 4B and SM for detail) by the electron energyfiltered diffraction (eEFD) method (30). A total of 46 datasets were merged, and the highresolution limit was determined to be $0.85 \AA$. The initial structure was successfully solved with a space group $\mathrm{P}_{2}$ by molecular replacement, starting from the structure of $\mathbf{1 - C l}$, and direct phasing also gave the same solution. As shown in Figure 4C, the molecular structure of 1-H was confirmed as expected.

In the crystal, four crystallographically nonequivalent molecules (1-H-a-d in Figure 4D) were found. Two of the four (1-H-a and 1-H-b) have the same helical chirality around the sevenmembered-ring moieties $(P M P)$, whereas the other two (1-H-c and 1-H-d) have the opposite chirality $(M P M)$. Continuous $\pi-\pi$ stacking of alternating $\mathbf{1 - H}-\mathbf{a}$ and $\mathbf{1 - H}-\mathbf{b}$ units (Figure 4E) results in the formation of homochiral nanofibers that mesh in the needle-shaped microcrystals with adjacent nanofibers composed entirely of molecules having the opposite chirality (1-H-c and 1-Hd, Figure 4F). Figures $4 \mathrm{G}$ and $4 \mathrm{H}$ provide a closer look at the molecular alignment structures within a single nanofiber. Each nanofiber comprises a double helix structure wherein 1-H molecules of the same chirality are stacked with $45^{\circ}$ twist angles. Judging from the result that the diameters of a single nanofiber $(2.8 \mathrm{~nm}$, Figure $4 \mathrm{G})$ matches the height of the thin nanofiber found in the AFM measurement $(2.8 \mathrm{~nm}$, Figure $3 \mathrm{~F})$, this supramolecular double helix alignment beautifully accounts for the structure of the nanofibers found in the gel state. The pitch length of the helix is $3.1 \mathrm{~nm}$, and 8 molecules constitute a helical repeat unit (Figure $4 \mathrm{H}$ ). These findings clearly demonstrate the power of 3D electron crystallography for the structural determination of nanometer-sized molecular alignments, even for cases in which single crystals suitable for x-ray crystallography cannot be obtained. 

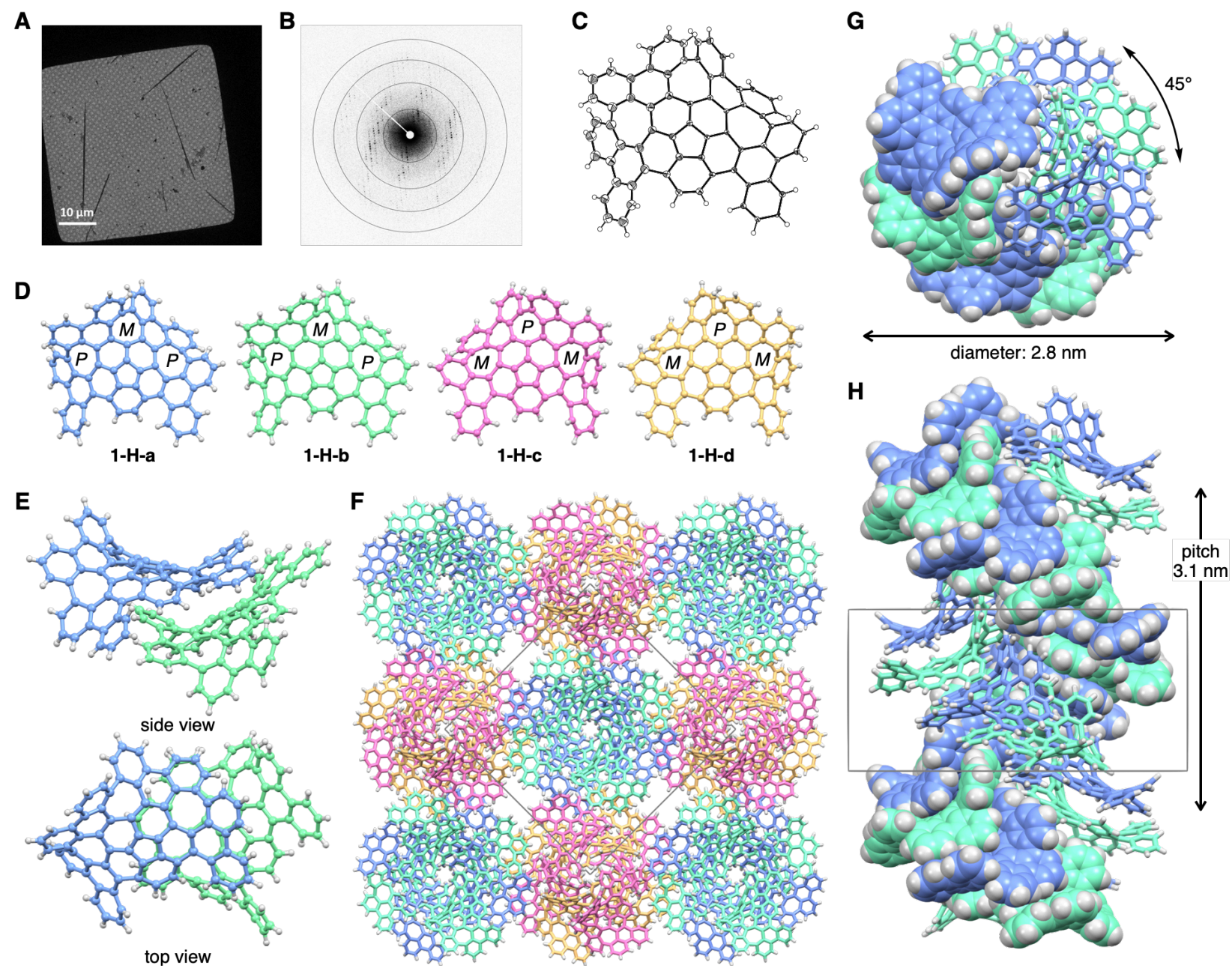

Figure 4. Structure of the nanofibers of 1-H revealed by 3D electron crystallography. (A) A cryo-EM image of fibrous microcrystals distributed on a holey carbon film. (B) Representative frames of electron diffraction patterns in a rotation angle of $-4^{\circ} \sim-3^{\circ}$. Concentric circles indicate $3.0,1.5,1.0$, and $0.8 \AA$ resolution rings. (C) ORTEP drawing of $\mathbf{1 - H}$ with $50 \%$ probability determined by eEFD. (D) Four crystallographically inequivalent molecules of 1-H; helical chiralities $(P$ or $M)$ around the seven-membered-ring moieties are indicated. (E) A $\pi-\pi$ stacking mode of 1-H-a and 1-H-b. (F) Packing structure of 1-H along $c$ axis; a unit cell is shown in gray. (G) A double-helix nanofiber with its diameter and twist angle. (H) A double-helix nanofiber with its helix pitch; a unit cell is shown in gray.

To prepare supramolecular stacks of nanographenes, chemists have historically relied heavily on the use of assembly-assisting substituents. We have now discovered the first-in-class polycyclic $\pi$-system, consisting solely of $\mathrm{sp}^{2}$-hybridized carbon atoms, that spontaneously stacks without any assembly-assisting substituents. Our new, well-designed nanographene with negative curvature self-assembles efficiently with offset $\pi-\pi$ stacking $\left(45^{\circ}\right.$ twist angles in the present case), ultimately creating beautiful, double-helix, supramolecular nanofibers. Based on this discovery and its revelation of a new guiding principle in supramolecular self-assembly, we expect that a number of negatively curved nanographenes can be developed for new applications in materials science and 
biology. Moreover, this work not only reports the discovery of an all-sp ${ }^{2}$-carbon supramolecular $\pi$-organogelator with negative curvature, but it also showcases the power of $3 \mathrm{D}$ electron crystallography for the structural determination of submicrometer-sized hydrocarbon molecular assemblies. This ability to elucidate the layered structures adopted by negatively curved nanographenes at the molecular level will accelerate the future development of carbon-based materials science and technology.

\section{References and Notes:}

1 A. Hirsch, The era of carbon allotropes. Nat. Mater. 9, 868-871 (2010).

2 A. K. Geim, K. S. Novoselov, The rise of graphene. Nat. Mater. 6, 183-191 (2007).

3 J. M. Tarascon, M. Armand, Issues and challenges facing rechargeable lithium batteries. Nature 414, 359-367 (2001).

4 T. Aida, E. W. Meijer, S. I. Stupp, Functional supramolecular polymers. Science 335, 813817 (2012).

5 F. Ishiwari, Y. Shoji, T. Fukushima, Supramolecular scaffolds enabling the controlled assembly of functional molecular units. Chem. Sci. 9, 2028-2041 (2018).

6 F. J. M. Hoeben, P. Jonkheijm, E. W. Meijer, A. P. H. J. Schenning, About supramolecular assemblies of $\pi$-conjugated systems. Chem. Rev. 105, 1491-1546 (2005).

7 P. Herwig, C. W. Kayser, K. Müllen, H. W. Spiess, Columnar mesophases of alkylated hexaperi-hexabenzocoronenes with remarkably large phase widths. Adv. Mater. 8, 510-513 (1996).

8 D. Miyajima, K. Tashiro, F. Araoka, H. Takezoe, J. Kim, K. Kato, M. Takata, T. Aida, Liquid crystalline corannulene responsive to electric field. J. Am. Chem. Soc. 131, 44-45 (2009).

9 T. Nagano, K. Nakamura, Y. Tokimaru, S. Ito, D. Miyajima, T. Aida, K. Nozaki, Functionalization of azapentabenzocorannulenes by fivefold $\mathrm{C}-\mathrm{H}$ borylation and crosscoupling arylation: Application to columnar liquid-crystalline materials. Chem. Eur. J. 24, 14075-14078 (2018).

10 Y. Shoji, T. Kajitani, F. Ishiwari, Q. Ding, H. Sato, H. Anetai, T. Akutagawa, H. Sakurai, T. Fukushima, Hexathioalkyl sumanenes: An electron-donating buckybowl as a building block for supramolecular materials. Chem. Sci. 8, 8405-8410 (2017).

11 K. Kawasumi, Q. Zhang, Y. Segawa, L. T. Scott, K. Itami, A grossly warped nanographene and the consequences of multiple odd-membered-ring defects. Nat. Chem. 5, 739-744 (2013).

12 K. Kato, Y. Segawa, L. T. Scott, K. Itami, Synthesis, properties, and packing structures of corannulene-based $\pi$-systems containing heptagons. Chem. Asian J. 10, 1635-1639 (2015).

13 H.-A. Lin, Y. Sato, Y. Segawa, T. Nishihara, N. Sugimoto, L. T. Scott, T. Higashiyama, K. Itami, A water-soluble warped nanographene: Synthesis and applications for photoinduced cell death. Angew. Chem., Int. Ed. 57, 2874-2878 (2018).

14 K. Kato, H.-A. Lin, M. Kuwayama, M. Nagase, Y. Segawa, L. T. Scott, K. Itami, Two-step synthesis of a red-emissive warped nanographene derivative via a ten-fold $\mathrm{C}-\mathrm{H}$ borylation. Chem. Sci. 10, 9038-9041 (2019). 
15 H.-A. Lin, K. Kato, Y. Segawa, L. T. Scott, K. Itami, Synthesis and structural features of thiophene-fused analogues of warped nanographene and quintuple helicene. Chem. Sci. 10, 2326-2330 (2019).

16 M. N. Eliseeva, L. T. Scott, Pushing the Ir-catalyzed C-H polyborylation of aromatic compounds to maximum capacity by exploiting reversibility. J. Am. Chem. Soc. 134, 1516915172 (2012).

17 M. Grzybowski, K. Skonieczny, H. Butenschön, D. T. Gryko, Comparison of oxidative aromatic coupling and the Scholl reaction. Angew. Chem., Int. Ed. 52, 9900-9930 (2013).

18 M. Grzybowski, B. Sadowski, H. Butenschön, D. T. Gryko, Synthetic applications of oxidative aromatic coupling-from biphenols to nanographenes. Angew. Chem., Int. Ed. 59, 2998-3027 (2020).

19 E. R. Draper, D. J. Adams, Low-molecular-weight gels: the state of the art. Chem 3, 390-410 (2017).

20 N. M. Sangeethaz, U. Maitra, Supramolecular gels: Functions and uses. Chem. Soc. Rev. 34, 821-836 (2005).

21 T. Kato, Y. Hirai, S. Nakaso, M. Moriyama, Liquid-crystalline physical gels. Chem. Soc. Rev. 36, 1857-1867 (2007).

22 S. S. Babu, V. K. Praveen, A. Ajayaghosh, Functional $\pi$-gelators and their applications. Chem. Rev. 114, 1973-2129 (2014).

23 P. Terech, R. G. Weiss, Low molecular mass gelators of organic liquids and the properties of their gels. Chem. Rev. 97, 3133-3159 (1997).

24 D. J. Abdallah, R. G. Weiss, Organogels and low molecular mass organic gelators. Adv. Mater. 12, 1237-1247 (2000).

25 P. Dastidar, Supramolecular gelling agents: Can they be designed? Chem. Soc. Rev. 37, 26992715 (2008).

26 A. Dawn, T. Shiraki, S. Haraguchi, S.-I. Tamaru, S. Shinkai, What kind of "soft materials" can we design from molecular gels? Chem. Asian J. 6, 266-282 (2011).

27 A. Ajayaghosh, V. K. Praveen, $\pi$-Organogels of self-assembled $p$-phenylenevinylenes: Soft materials with distinct size, shape, and functions. Acc. Chem. Res. 40, 644-656 (2007).

28 A. Ajayaghosh, V. K. Praveen, C. Vijayakumar, Organogels as scaffolds for excitation energy transfer and light harvesting. Chem. Soc. Rev. 37, 109-122 (2008).

29 Z. Zhao, J. W. Y. Lam, B. Z. Tang, Self-assembly of organic luminophores with gelationenhanced emission characteristics. Soft Matter 9, 4564-4579 (2013).

30 K. Yonekura, T. Ishikawa, S. Maki-Yonekura, A new cryo-EM system for electron 3D crystallography by eEFD. J. Struct. Biol. 206, 243-253 (2019).

\section{Acknowledgments:}

This work was supported by the ERATO program from JST (JPMJER1302 to K.I.), the Funding Program for KAKENHI from MEXT (JP1905463 to K.I.; JP16K05771, JP19H02701, and JP19K22183 to Y.S.), a grant-in-aid for Scientific Research on Innovative Areas " $\pi$-Figuration" 
from the JSPS (JP17H05149 to Y.S.), the Cyclic Innovation for Clinical Empowerment (CiCLE) from the Japan Agency for Medical Research and Development, AMED (to K.Y.), Toyoaki Scholarship Foundation (to Y.S.), Daiko Foundation (to Y.S.), and the US National Science Foundation (CHE-1 149096 to L.T.S.). K.K. thanks the IGER Program in Green Natural Sciences (Nagoya University) and the JSPS for a fellowship for young scientists. The authors thank S. Seki, Y. Tsutsui T. Uchihashi, S. Ogi, H. Sakamoto, J. Shim, E. Ito, J. Shirasaki, K. Yamanoue, M. Toya, N. Yasuda, and RIGAKU Co. for fruitful discussions and support with the measurements. ITbM is supported by the World Premier International Research Center Initiative (WPI), Japan.

\section{Supplementary Materials:}

$10 \quad$ Materials and Methods

Figures S1-S7

Tables S1-S2

References (31-47) 